\title{
PATH ALGEBRAIC AHP EIGENVECTOR BASED ON MAX AND MIN OPERATORS
}

\author{
Masaaki Shinohara \\ Nihon University \\ Izumi-chou, Narashino \\ Chiba 275-8575, Japan \\ E-mail: shinohara.masaaki@nihon-u.ac.jp
}

\begin{abstract}
The dynamic averaging process or DAP, which is supposed to simulate the priority weight determination process on the cognitive layer in a human mind is generalized from the conventional arithmetic mean averaging to the path algebraic MAX and MIN operations. Conventional DAP with the arithmetic mean averaging, or Arithmetic-DAP, employs at each iteration a decision making among the items compared, where a harmony among different opinions is considered important, and two opinions are summed up, divided by two, and then made into one compromising opinion. In this paper, we propose a new scheme of DAP with MAX or MIN operator, MAX-DAP or MIN-DAP, which employs at each iteration a severe decision making among the compared items; say, with the worst case analysis, two opinions are compared and the maximum of the two is chosen in case of the cost weight, which is a non- compromising opinion. The path algebraic formulation of the AHP EIGEN weight vector is presented and its numerical examples are shown by using $4 \times 4$ comparison matrices. Some periodic oscillation phenomena are observed in the dynamic performance of MAX-DAP when comparison matrices are inconsistent. Some implications of using the non-compromising solutions derived from the proposed MAX-DAP or MIN-DAP are suggested, in a process of the Analytic Hierarchy Process.
\end{abstract}

Keywords: path algebra, eigenvector in AHP, MAX operator, MIN operator, dynamic averaging process or DAP, compromising solution, non-compromising solution, periodic oscillation

\section{Introduction}

The dynamic averaging process or DAP, which is supposed to simulate the priority weight determination process on the cognitive layer in a human mind, from a set of pairwise comparison judgments, is generalized from the conventional arithmetic mean averaging to the path algebraic MAX and MIN operations. Conventional DAP with the arithmetic mean averaging, or Arithmetic-DAP, employs at each iteration a decision making among the items compared, where a harmony among different opinions (different values of the priority weight of an item viewed from the comparing items) is considered important, and two (or N) opinions are summed up, divided by two $($ or $\mathrm{N})$, and then made into one, which is nothing but the arithmetic mean. Even when the set of pairwise comparison judgments is inconsistent, Arithmetic-DAP converges to a stationary EIGEN weight vector, when the weight vector is normalized so that the sum of each element in a vector is unity. In this paper, we propose a new scheme of DAP with MAX or MIN operator, MAX-DAP or MIN-DAP, which employs at each iteration a severe decision making among the compared items; say, with the worst case analysis, two(or $\mathrm{N}$ ) opinions are compared and, instead of summing them up, the minimum among them is chosen in case of the benefit weight and the maximum among them is chosen in case of the cost weight.

In Chapter 2, the path algebra $\mathrm{P}(\oplus, \otimes)$ is introduced and the eigenvalue problem on the path algebra $\mathrm{P}(\oplus$ ,() is studied. In Chapter 3, a new scheme of DAP with MAX or MIN operator, MAX-DAP or MINDAP, is proposed and its decision making attitude when comparing different opinions (different values of the priority weight of an item viewed from the comparing items) is discussed. In Chapter 4, it is shown that some periodic oscillations are observed in the numerical examples of the proposed MAX-DAP. 


\section{Path algebra $P(\oplus, \bigotimes)$ and its eigenvalue problem}

Consider a linear matrix equation (1), where $A$ is an $n \times n$ coefficient matrix, $X$ is an $n \times n$ unknown variable matrix, and $\mathrm{B}$ can be the $\mathrm{n} \times \mathrm{n}$ unit matrix $\mathrm{I}$.

$\mathrm{X}=\mathrm{A} \cdot \mathrm{X}+\mathrm{B}(1)$

Theory of linear matrix algebra tells us that the under certain conditions the equation (1) has a solution (2), or the solution (2) converges.

$\mathrm{X}=\sum_{\mathrm{k}=0}^{\mathrm{m}} \mathrm{A}^{\mathrm{k}} \cdot \mathbb{B}(2)$

A path algebra $\mathrm{P}(\oplus, \otimes)$ can be defined, in a narrow sense, as that of the linear matrix algebra as in (1) and (2) where the ordinary addition operator + and the ordinary multiplication operator $X$ are replaced with the generalized addition operator $\oplus$ and the generalized multiplication operator $\otimes$. Since the $(i, j)$ th element of $A^{\text {kx }}$ in (2) means "the number of k-step paths, or the sum of k-step chain product gains, from node $\mathrm{i}$ to node $\mathrm{j}$ in a directed network with weight $\mathrm{A}$ ", this linear matrix algebra with the operators $\oplus$ and $\otimes$ is called "path algebra $\mathrm{P}(\oplus, \otimes)$, or $\mathrm{P}(\mathrm{A}, \oplus, \otimes)$ ". By setting $\oplus=$ min and $\otimes=+$, the shortest path problem is formulated by the matrix equation (3); on the basis of this formulation, various algorithms can be conceived to solve the shortest path problem (ex. Shinohara, 1973).

The path algebraic problem is generally formulated by (3), where B can be the unit matrix $\mathrm{I}(\oplus, \otimes)$ for the path algebra $\mathrm{P}(\oplus, \otimes)$ and the matrix operators $\boxplus$ and $\otimes$ are defined by (4) and (5), respectively. Here, the unit matrix $\mathrm{I}(\oplus, \otimes)$ has the identities of the algebra $\mathrm{P}(\oplus, \otimes)$ at its diagonal elements and the zeros of the algebra $\mathrm{P}(\oplus, \otimes)$ at its non-diagonal elements.

$\mathrm{X}=(\mathrm{A} \otimes \mathrm{X}) \oplus \mathrm{B} \quad(3)$

$\mathrm{c}_{\mathrm{ij}}=\mathrm{d}_{\mathrm{ij}} \oplus \mathrm{e}_{\mathrm{ij}}$ for the matrix operation $\mathrm{C}=\mathrm{D} \boxplus \mathrm{E} \quad$ (4)

$\mathrm{c}_{\mathrm{ij}}=\left(\mathrm{d}_{\mathrm{i} 1} \otimes \mathrm{e}_{\mathrm{ij}}\right) \oplus\left(\mathrm{d}_{\mathrm{i} 2} \otimes \mathrm{e}_{2 \mathrm{j}}\right) \oplus \cdots \oplus\left(\mathrm{d}_{\mathrm{in}} \otimes \mathrm{e}_{\mathrm{ni}}\right) \quad$ for the matrix operation $\mathrm{C}=\mathrm{D} \otimes \mathrm{E} \quad$ (5)

Under certain conditions the matrix equation (3) is expected to have a solution of the form (6), where $A^{k}$ is defined by (7).

$\mathrm{X}=\left(\Sigma_{k=0}^{\infty} \boxplus \mathrm{A}^{\mathrm{k}}\right) \otimes \mathrm{B} \quad(6)$

$\mathrm{A}^{\mathrm{k}}=\mathrm{A} \bigotimes(\mathrm{A} \bigotimes(\mathrm{A} \bigotimes(\ldots \bigotimes \mathrm{A}) \ldots))$

Next, consider the case where the solution (2) or (6) diverges (just in the case of applying the l'Hôpital's rule). By choosing $\mathrm{B}$ as the zero matrix with all its elements being the zeros of the algebra $\mathrm{P}(\oplus, \otimes)$ and regarding (1) or (3) as an iteration, an iterative process (8) or (9) is obtained.

$\mathrm{X}(\mathrm{t}+1)=\mathrm{A} \cdot \mathrm{X}(\mathrm{t})(8)$

$\mathrm{X}(\mathrm{t}+1)=\mathrm{A} \bigotimes \mathrm{X}(\mathrm{t})(9)$

The corresponding matrix-form eigenvalue problems can be formally formulated by (10) and (11), where $\mathrm{L}$ is the diagonal eigenvalue matrix defined by (12).

$\mathrm{A} \cdot \mathrm{X}=\mathrm{L} \cdot \mathrm{X}$

$\mathrm{A} \otimes \mathrm{X}=\mathrm{L} \otimes \mathrm{X}$

$\mathrm{L}=\operatorname{diag}\left(\lambda_{1}, \lambda_{2}, \ldots, \lambda_{\mathrm{n}}\right)$

\section{Dynamic averaging process with MAX or MIN operator}

First, conventional dynamic averaging process, the Arithmetic-DAP, is explained by an 4-item completeinformation example (Shinohara, 2011); the updating formula of the Arithmetic-DAP is given by (13), or generally by (14), where $\mathrm{N}$ is the number of the items compared.

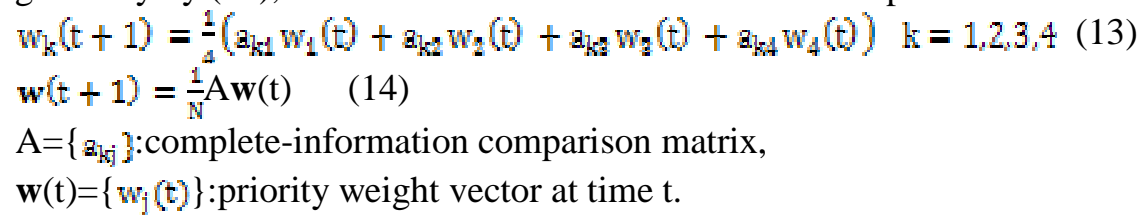


The updating formula (13)(or (14)) means that the four (or N) opinions about evaluating item $\mathrm{k}$ from the viewpoint of item $\mathrm{j}, \mathrm{a}_{\mathrm{j}} \times \mathrm{w}_{\mathrm{j}}(\mathrm{j}=1,2,3,4)$, are summed up, divided by four (or $\mathrm{N}$ ), and then made into a new opinion about evaluating item $\mathrm{k}(\mathrm{k}=1,2,3,4)$.

Next, consider the MAX-DAP, whose updating formula is given by (15), or by (16).

$\mathrm{w}_{\mathrm{k}}(\mathrm{t}+1)=\max \left\{\mathrm{a}_{\mathrm{k} 1} \mathrm{w}_{1}(\mathrm{t}), \mathrm{a}_{\mathrm{k} 2} \mathrm{w}_{2}(\mathrm{t}), \mathrm{a}_{\mathrm{k} 2} \mathrm{w}_{\mathrm{g}}(\mathrm{t}), \mathrm{a}_{\mathrm{k} 4} \mathrm{w}_{4}(\mathrm{t})\right\} \quad(15)$

$\mathbf{w}(\mathrm{t}+1)=\mathrm{A} \bigotimes \mathbf{w}(\mathrm{t})$

Here, the matrix operator $\bigotimes$ is defined by (5) on the path algebra $P(\oplus, \otimes)$ with $\oplus=\max$ and $\otimes=X$.

The updating formula (15) (or(16)) means that the four (or $\mathrm{N}$ ) opinions about evaluating item $\mathrm{k}$ from the viewpoint of item $\mathrm{j}, \mathbf{a}_{\mathrm{kj}} \times \mathrm{w}_{\mathrm{j}}(\mathrm{j}=1,2,3,4)$, are compared, the maximum among the four (or $\mathrm{N}$ ) opinions chosen, and then made into a new opinion about evaluating item $\mathrm{k}(\mathrm{k}=1,2,3,4)$.

Finally, consider the MIN-DAP, whose updating formula is given by (17), or by (18).

$w_{k}(t+1)=\min \left\{a_{k 1} w_{1}(t), a_{k 2} w_{2}(t), a_{k 3} w_{3}(t), a_{k 4} w_{4}(t)\right\}$

$\mathbf{w}(\mathrm{t}+1)=\mathrm{A} \otimes \mathbf{w}(\mathrm{t})$

Here, the matrix operator $\bigotimes$ is defined by (5) on the path algebra $P(\oplus, \otimes)$ with $\oplus=\min$ and $\otimes=X$.

The updating formula(17)(or(18)) means that the four (or $\mathrm{N}$ ) opinions about evaluating item $\mathrm{k}$ from the viewpoint of item $\mathrm{j}, \mathrm{a}_{\mathrm{kj}} \times \mathrm{w}_{\mathrm{j}}(\mathrm{j}=1,2,3,4)$, are compared, the minimum among the four (or $\mathrm{N}$ ) opinions chosen, and then made into a new opinion about evaluating item $\mathrm{k}(\mathrm{k}=1,2,3,4)$.

The three decision making attitudes employed at each iteration of a DAP are summarized in Table 1.

Table1: Three DAP's and their decision making attitudes

\begin{tabular}{|l|l|l|l|}
\hline Averaging method & Updating & Decision making attitude \\
\cline { 3 - 4 } employed in DAP & formula & Benefit weight & Cost weight \\
\hline $\begin{array}{l}\text { Arithmetic mean } \\
\text { averaging }\end{array}$ & $(13),(14)$ & compromising & compromising \\
\hline Max operation & $(15),(16)$ & $\begin{array}{l}\text { the best case } \\
\text { (optimistic) }\end{array}$ & $\begin{array}{l}\text { the worst case } \\
\text { (pessimistic) }\end{array}$ \\
\hline Min operation & $(17),(18)$ & $\begin{array}{l}\text { the worst case } \\
\text { (pessimistic) }\end{array}$ & $\begin{array}{l}\text { the best case } \\
\text { (optimistic) }\end{array}$ \\
\hline
\end{tabular}

\section{Numerical examples and periodical oscillations observed in MAX-DAP}

The performance of MAX-DAP is numerically examined with $4 \times 4$ comparison matrices.

First, consider a consistent $4 \times 4$ matrix $C$ given by (19).

$\mathrm{C}=\left(\begin{array}{cccc}1 & 2 & 3 & 4 \\ 1 / 2 & 1 & 3 / 2 & 2 \\ 1 / 3 & 2 / 3 & 1 & 4 / 3 \\ 1 / 4 & 1 / 2 & 3 / 4 & 1\end{array}\right)$

Next, a non-reciprocal and hence inconsistent matrix $B$, given by (20), is made from the matrix $C$ by changing its $(1,2)$ th element value $c(1,2)=2$ to $b(1,2)=4$. 
$\mathrm{B}=\left(\begin{array}{cccc}1 & 4 & 3 & 4 \\ 1 / 2 & 1 & 3 / 2 & 2 \\ 1 / 3 & 2 / 3 & 1 & 4 / 3 \\ 1 / 4 & 1 / 2 & 3 / 4 & 1\end{array}\right)$

Finally, another inconsistent and non-reciprocal matrix A, given by (21), is made from the matrix B by changing its $(3,4)$ th and $(4,3)$ th element values to $\mathrm{a}(3,4)=\mathrm{a}(4,3)^{-1}=\mathrm{P}$.

$$
\mathrm{A}=\left(\begin{array}{cccc}
1 & 4 & 3 & 4 \\
1 / 2 & 1 & 3 / 2 & 2 \\
1 / 3 & 2 / 3 & 1 & \mathrm{P} \\
1 / 4 & 1 / 2 & \mathrm{P}^{-1} & 1
\end{array}\right)
$$

Figs. 1 and 2 show the MAX-DAP performances, or the normalized priority weights 1, 2, 3 and 4, for the consistent matrix $\mathrm{C}$ and the inconsistent matrix $\mathrm{B}$, respectively (see Appendix 1 for the raw data of the dynamic characteristics of Figs.1 and 2).

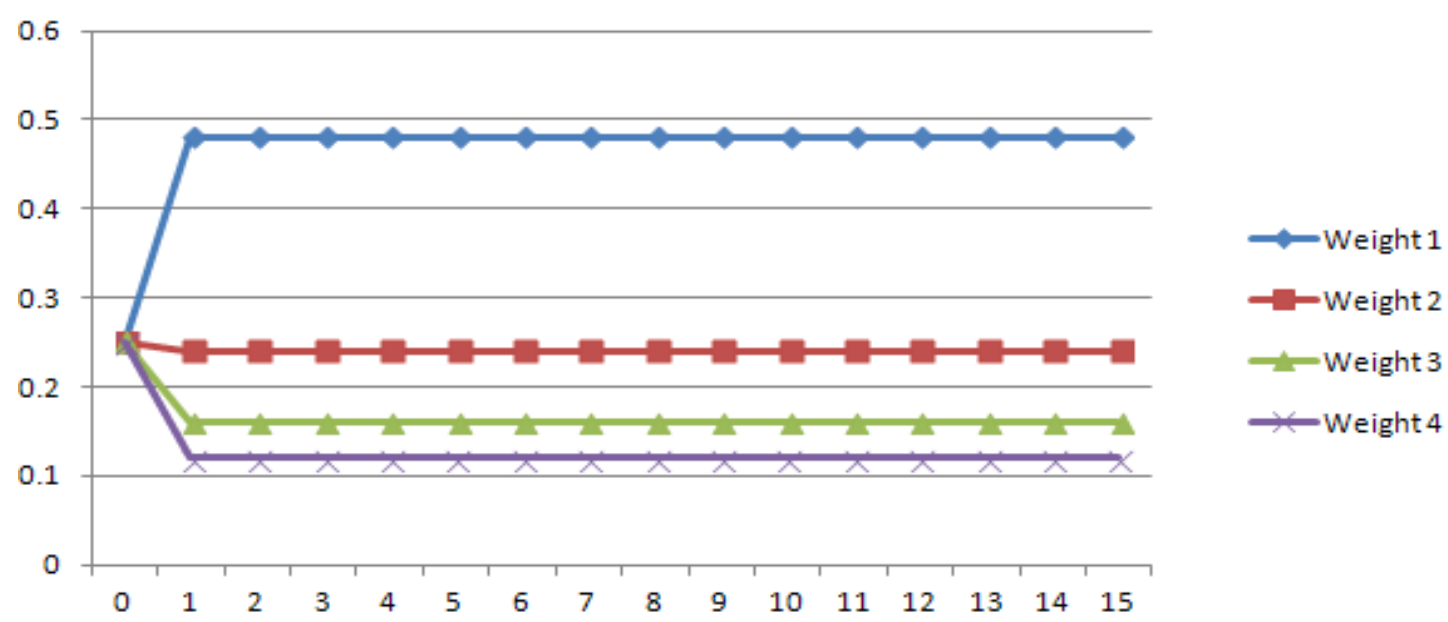

Fig.1: Dynamic characteristics of the normalized priority weight vector for matrix C.

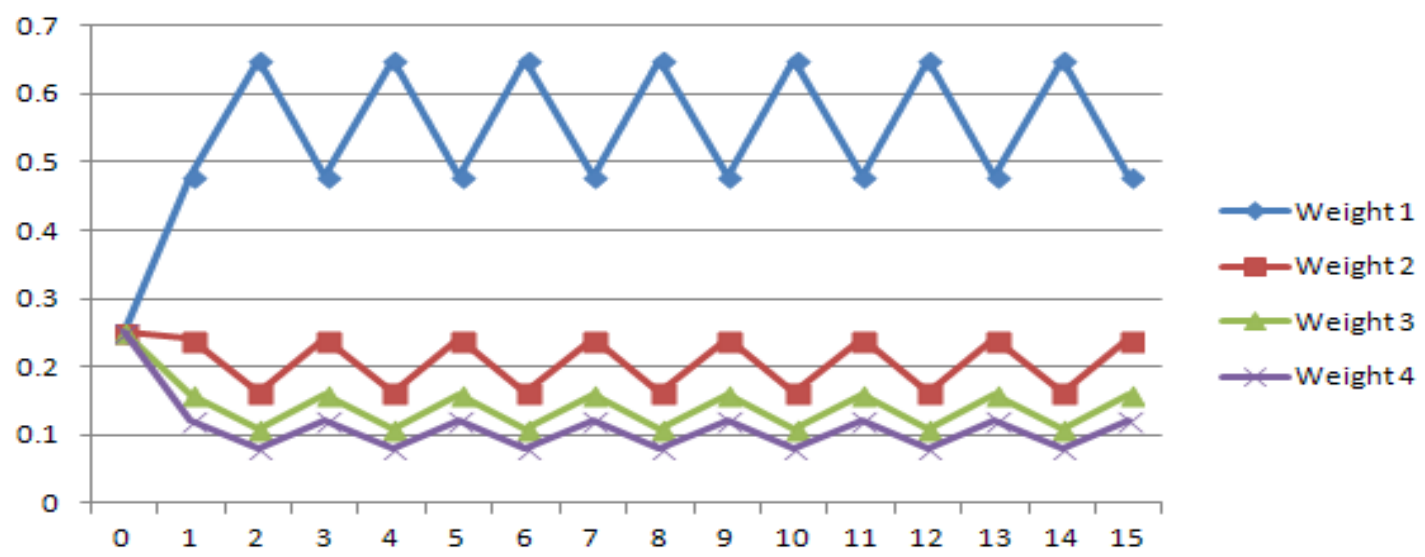

Fig.2: Dynamic characteristics of the normalized priority weight vector for matrix B. 
Figs. 3 and 4 show the MAX-DAP performances, or the normalized priority weights 1, 2, 3 and 4 , for the inconsistent matrix A with $\mathrm{P}=0.5$ and $\mathrm{P}=4$, respectively (see Appendix 1 for the raw data of the dynamic characteristics of Figs.3 and 4).

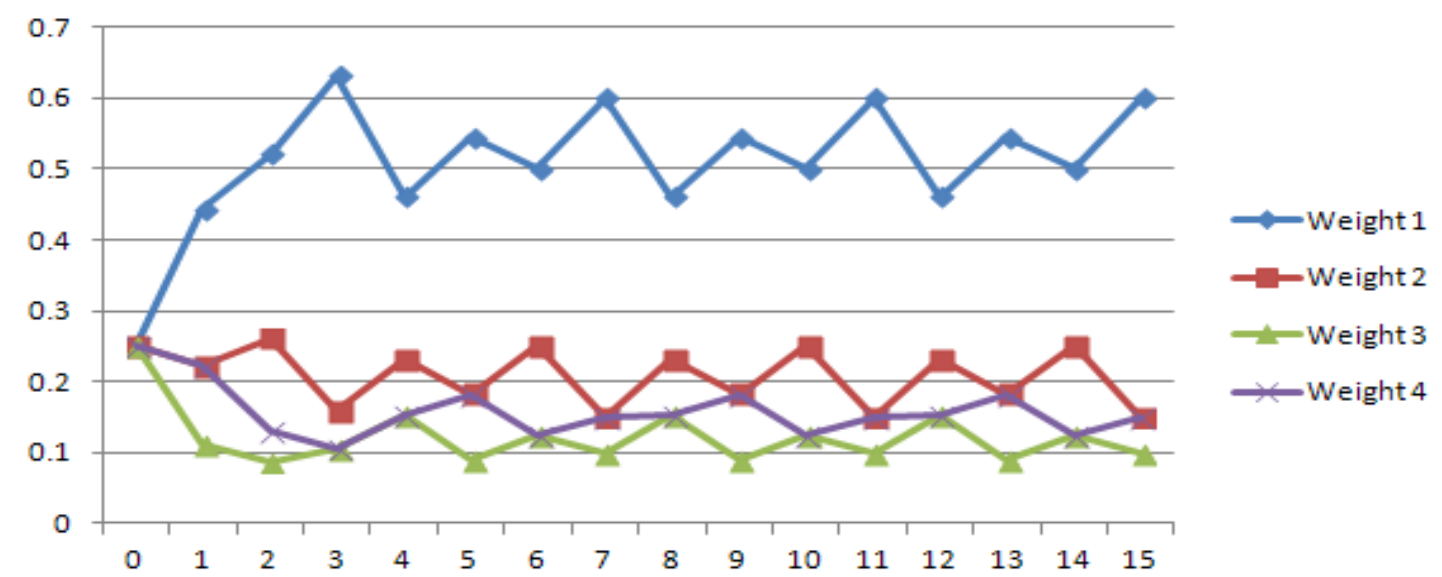

Fig.3: Dynamic characteristics of the normalized weight vector for matrix A with $\mathrm{P}=0.5$.

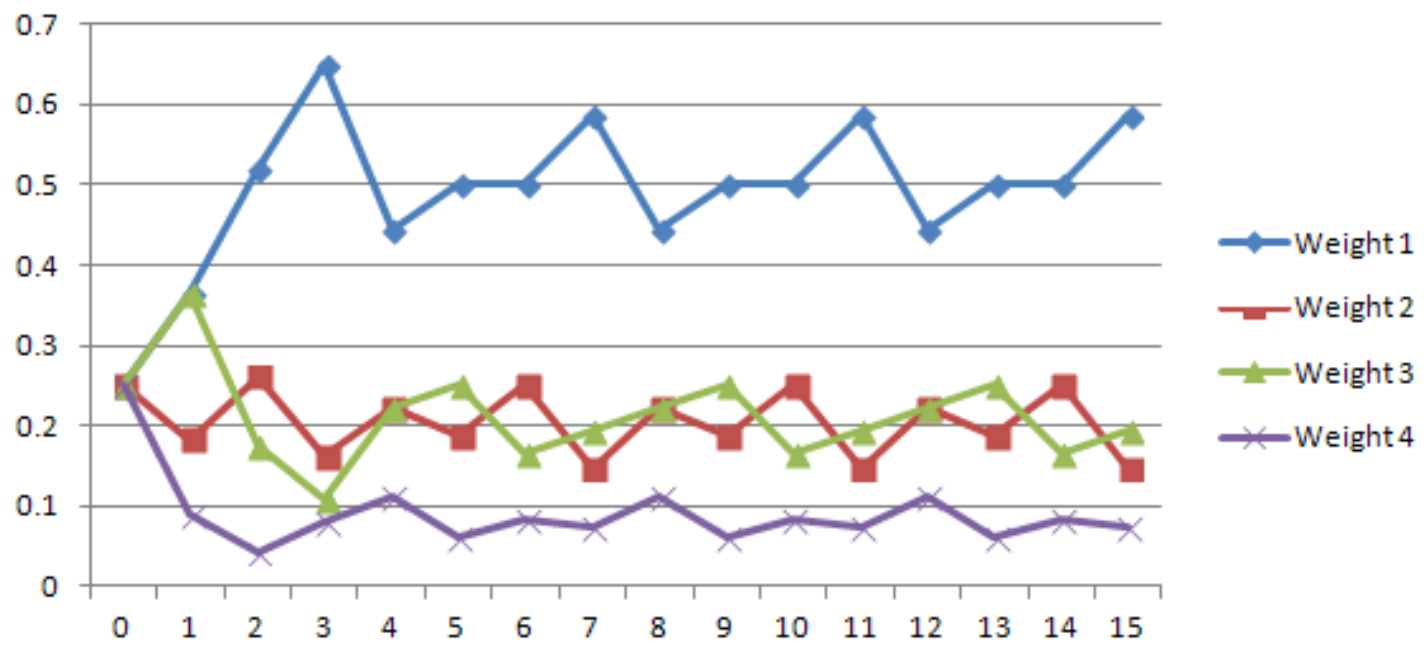

Fig.4: Dynamic characteristics of the normalized weight vector for matrix A with $\mathrm{P}=4$.

[Comment 1] As shown in Fig.1, the dynamic characteristics of the priority weight vector of MAX-DAP are stable for a consistent matrix, regardless of being normalized data or raw data (see also Fig.A1). Remember that, for any consistent matrix, the priority weight vector of the Arithmetic-DAP is also stable (Shinohara, 2011).

[Comment 2] As shown in Figs.2, 3 and 4, the priority weight vector of the MAX-DAP can be oscillating with some time period for inconsistent matrices.

[Comment 3] As for the non-reciprocal matrix $\mathrm{B}$, an oscillation of period $\mathrm{T}=2$ can be observed if $b(1,2) b(2,1)>1$ and an oscillation of period $T=2$ is not observed if $b(1,2) b(2,1) \leq 1$, by changing only the value of $b(1,2)$. It is interesting to notice that, when $b(1,2)=8 \quad(b(1,2) b(2,1)=4>1)$, no oscillation is observed(see Appendix 2 for the no-oscillation characteristics in this case), and that, even if $b(1,2) b(2,1) \leq 1$, oscillations of period lengths other than $T=2$ can be observed by changing the values of both $b(1,2)$ and $b(2,1)$ (see Appendix 3 for an oscillating characteristics of $T=3$ in this case).

[Comment 4] As for the inconsistent matrix A, oscillations of period $\mathrm{T}=4$ can be observed by appropriately setting the parameter $\mathrm{P}$, such as $\mathrm{P}=0.5$ and $\mathrm{P}=4$. Generally, oscillations of period $\mathrm{T}=2$ (such 
as in Fig.2) or $\mathrm{T}=3$ (such as in Fig.A8) can also be observed with $4 \times 4$ comparison matrices, but we cannot find an oscillation of period $\mathrm{T}=5$ or more with $4 \times 4$ comparison matrices.

\section{Conclusions}

A new scheme of dynamic averaging process, MAX-DAP or MIN-DAP, is proposed. Its numerical examples are shown and some periodic oscillation phenomena are observed in the dynamic performance of MAX-DAP when comparison matrices are inconsistent. In case of oscillation period $\mathrm{T}=2$, it means that two different weight vectors, or two different opinions, iteratively appear in our thinking process, and they are regarded as two extreme, or non-compromising, opinions. Before being satisfied with and instantly accepting the compromising solution derived from the conventional Arithmetic-DAP, isn't it worthwhile, as a process of the Analytic Hierarchy Process, discussing and arguing about the noncompromising solutions derived from the proposed MAX-DAP or MIN-DAP, in order to deepen an argument and reach a collaborative agreement.

\section{REFERENCES}

Shinohara, Masaaki (1973). The algebraic structure of network path problems and their algorithms (in Japanese), 1973 Fall Conference of Operations Research Society of Japan, 2-3-10, pp.137-138 (1973.11.8).

Saaty, T.L. (1980). The Analytic Hierarchy Process, McGraw-Hill, New York.

Shinohara, Masaaki (2011). AHP EIGENVECTOR VIA DYNAMIC PROCESS OF PAIRWISE COMPARISON AND AVERAGING, Proceedings of the International Symposium on the Analytic Hierarchy Process 2011, 144_019, (2011.6.16)

Shinohara, Masaaki and Ken Shinohara (2012). Theoretical Analysis of the Periodicity observed in MAX-DAP and MIN-DAP (in Japanese), Proceedings of the 45th Annual Conference of College of Industrial Technology, Nihon University, 2-74 (2012.12.1).

\section{Appendix 1: Unnormalized characteristics or raw data for Figs.1,2,3 and 4}




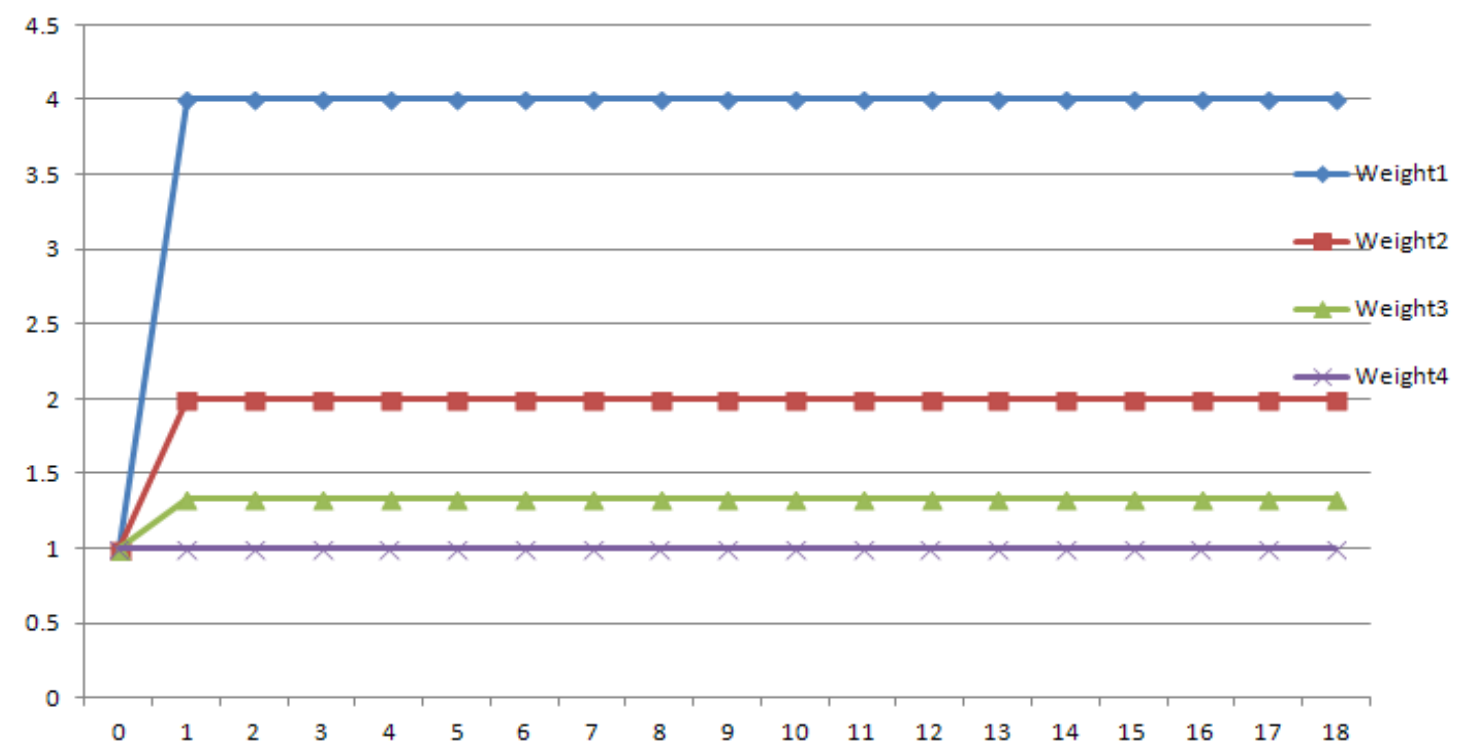

Fig.A1: Dynamic characteristics of the raw priority weight vector for matrix C.

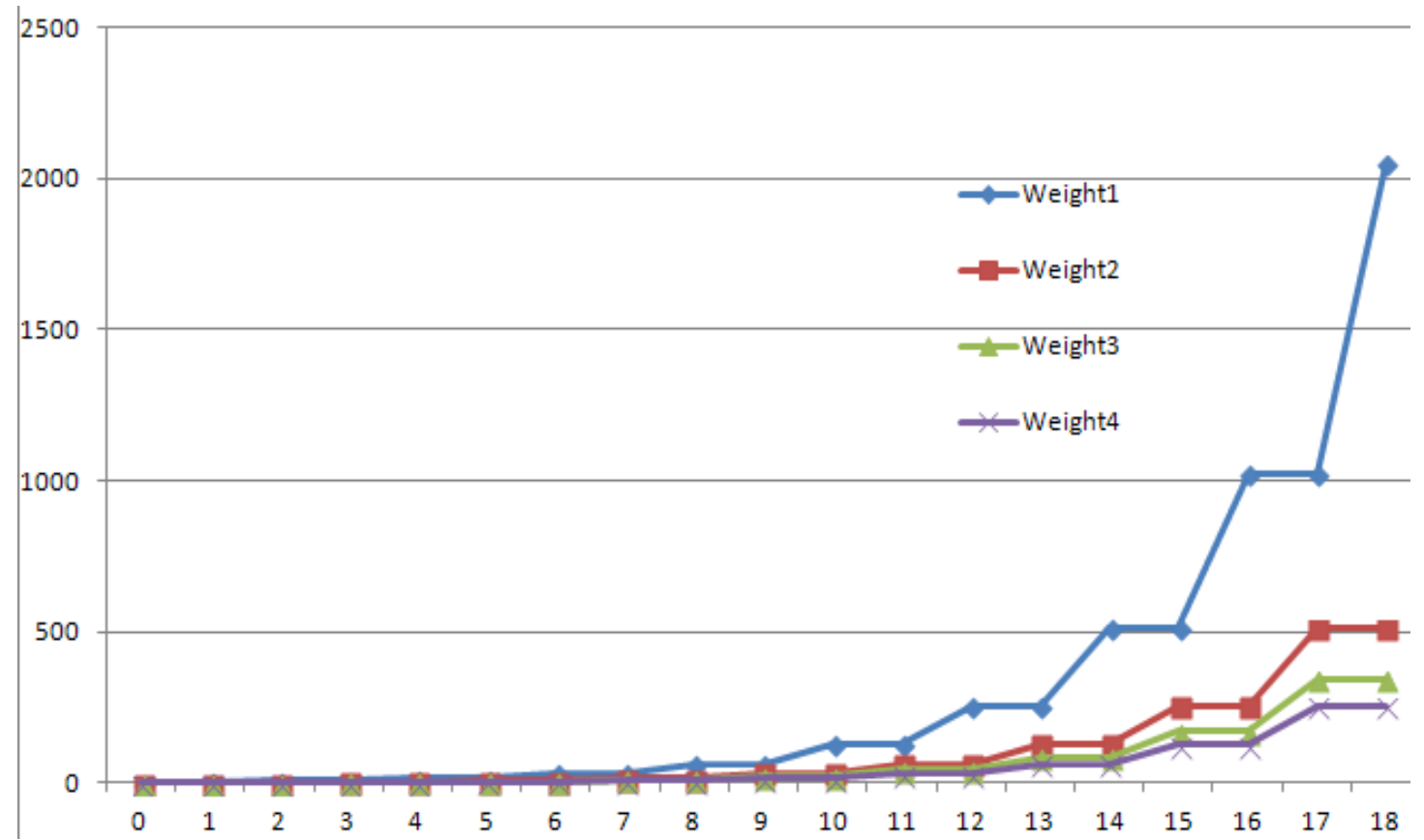

Fig.A2: Dynamic characteristics of the raw priority weight vector for matrix B. 
Proceedings of the International Symposium on the Analytic Hierarchy Process 2013

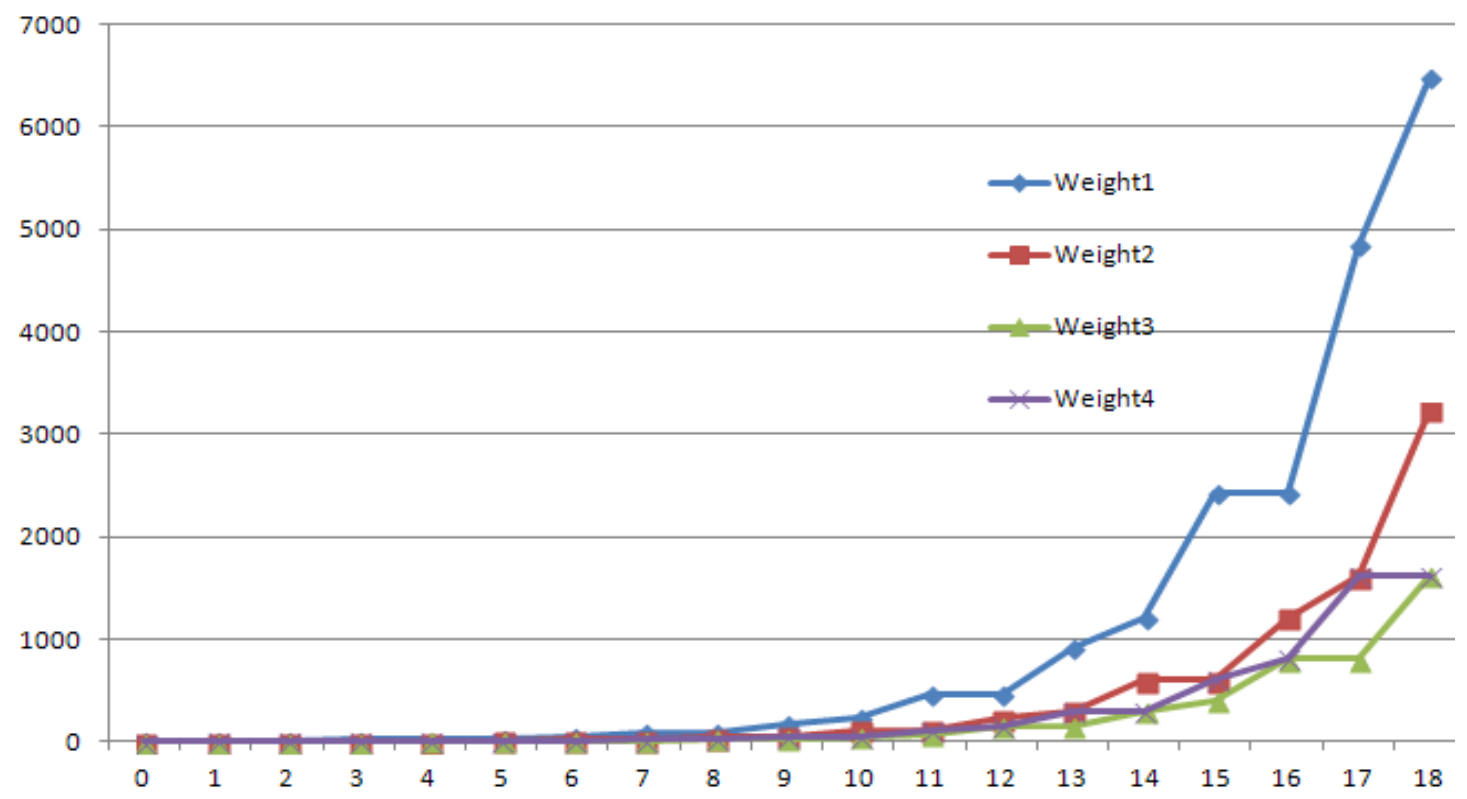

Fig.A3: Dynamic characteristics of the raw weight vector for matrix $A$ with $\mathrm{P}=0.5$.

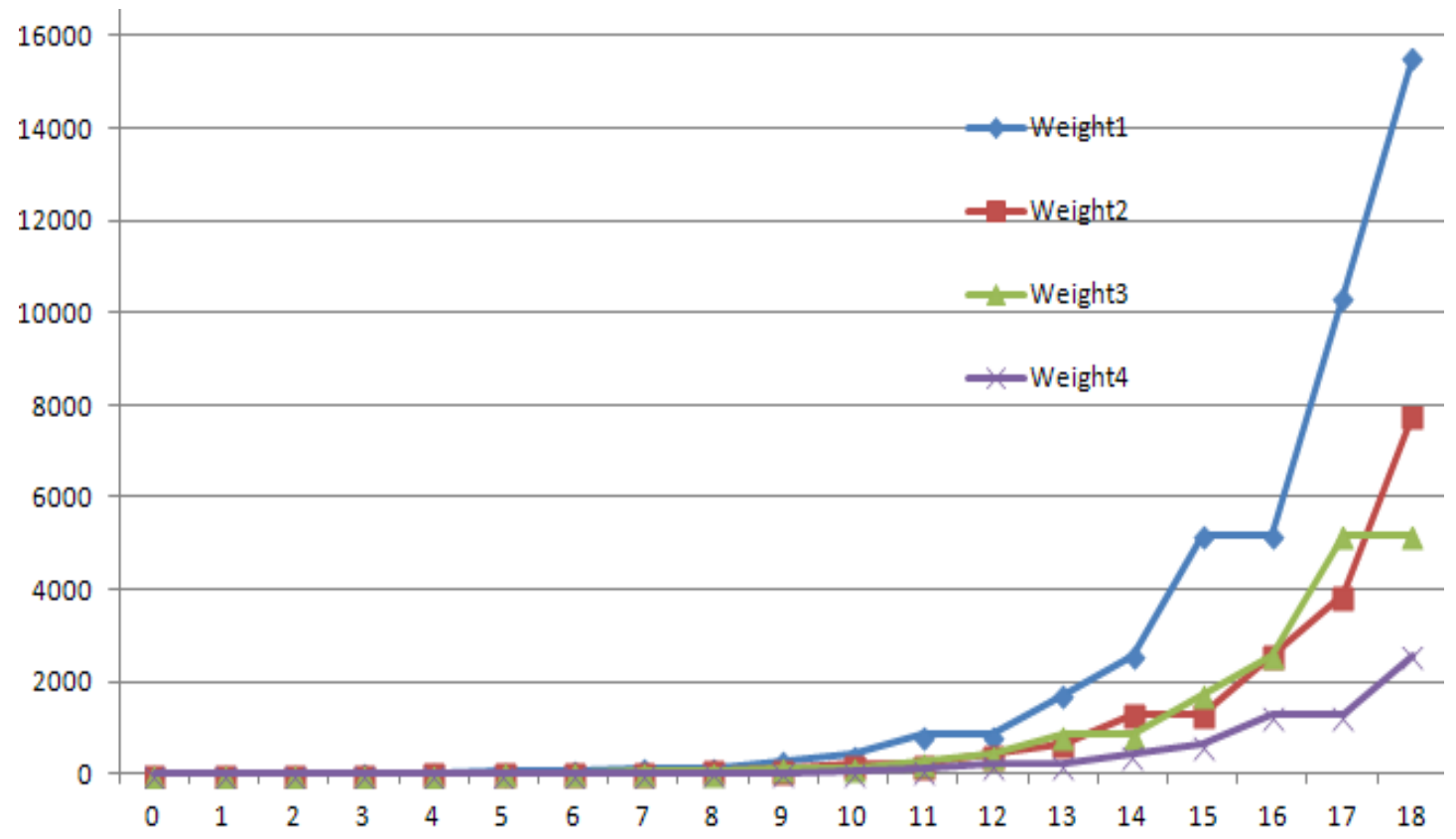

Fig.A4: Dynamic characteristics of the raw weight vector for matrix A with $\mathrm{P}=4$. 


\section{Appendix 2: No-oscillation characteristics for matrix $B_{1}$}

For the matrix $\mathrm{B}_{1}$ given by (A1), the raw and normalized characteristics are shown by Figs.A5 and A6.

$\mathrm{B}_{1}=\left(\begin{array}{cccc}1 & 8 & 3 & 4 \\ 1 / 2 & 1 & 3 / 2 & 2 \\ 1 / 3 & 2 / 3 & 1 & 4 / 3 \\ 1 / 4 & 1 / 2 & 3 / 4 & 1\end{array}\right)$

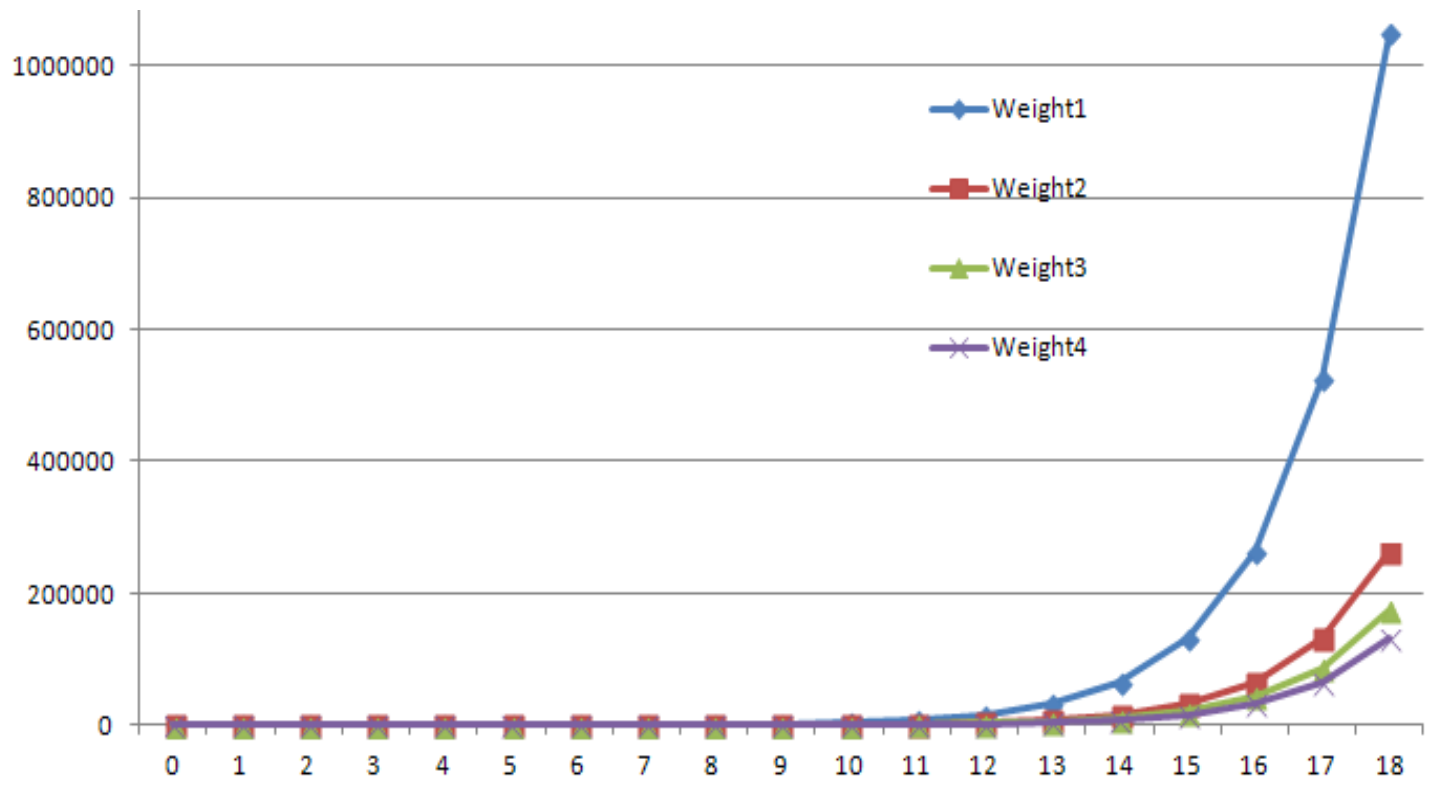

Fig.A5: Dynamic characteristics of the raw priority weight vector for matrix $\mathrm{B}_{1}$.

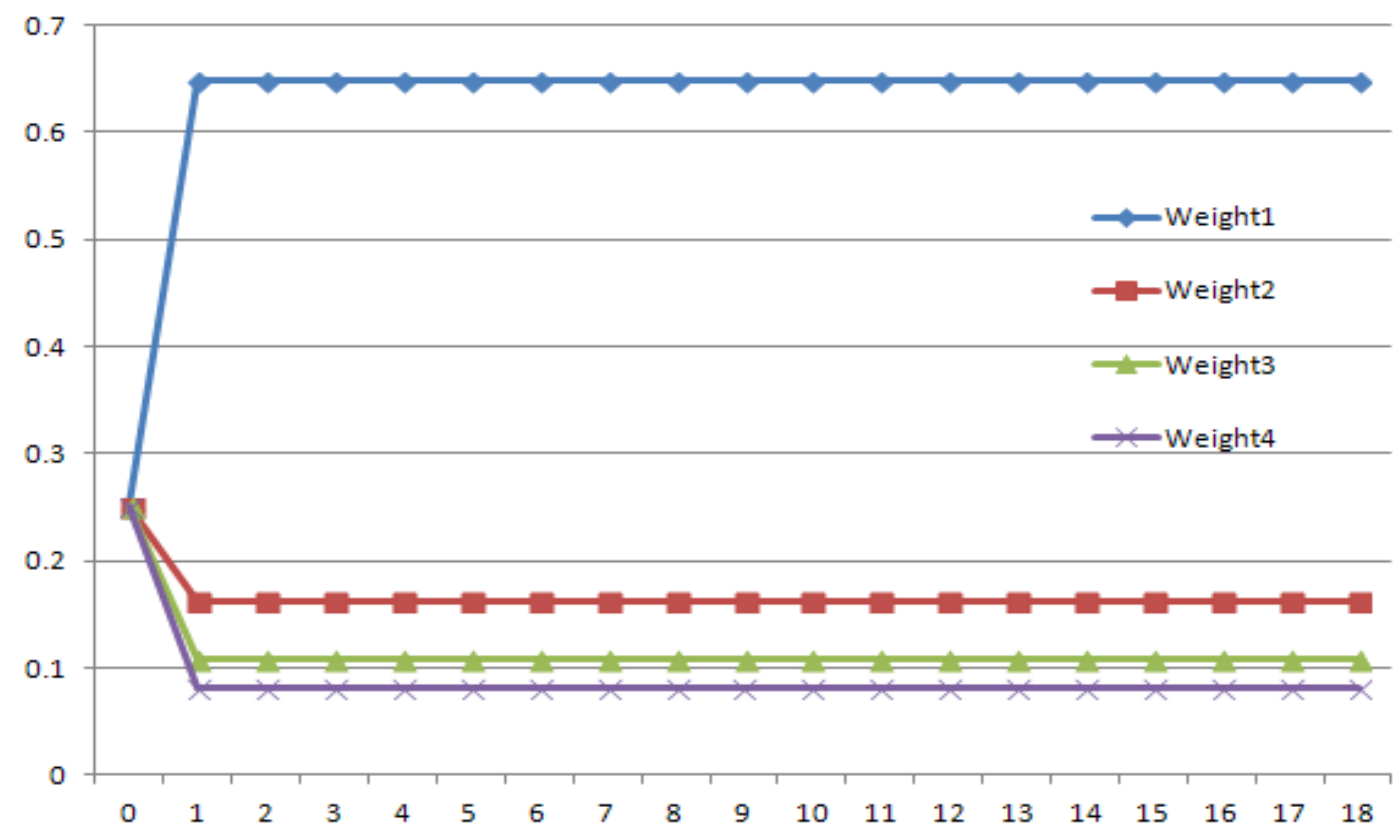

Fig.A6: Dynamic characteristics of the normalized priority weight vector for matrix $\mathrm{B}_{1}$. 


\section{Appendix 3: Oscillating characteristics of $\mathbf{T}=\mathbf{3}$ for matrix $\mathbf{B}_{\mathbf{2}}$}

For the matrix $\mathrm{B}_{2}$ given by (A2), the raw and normalized characteristics are shown by Figs. A7 and A8.

$\mathrm{B}_{2}=\left(\begin{array}{cccc}1 & 0.1 & 3 & 4 \\ 1 & 1 & 3 / 2 & 2 \\ 1 / 3 & 2 / 3 & 1 & 4 / 3 \\ 1 / 4 & 1 / 2 & 3 / 4 & 1\end{array}\right)$

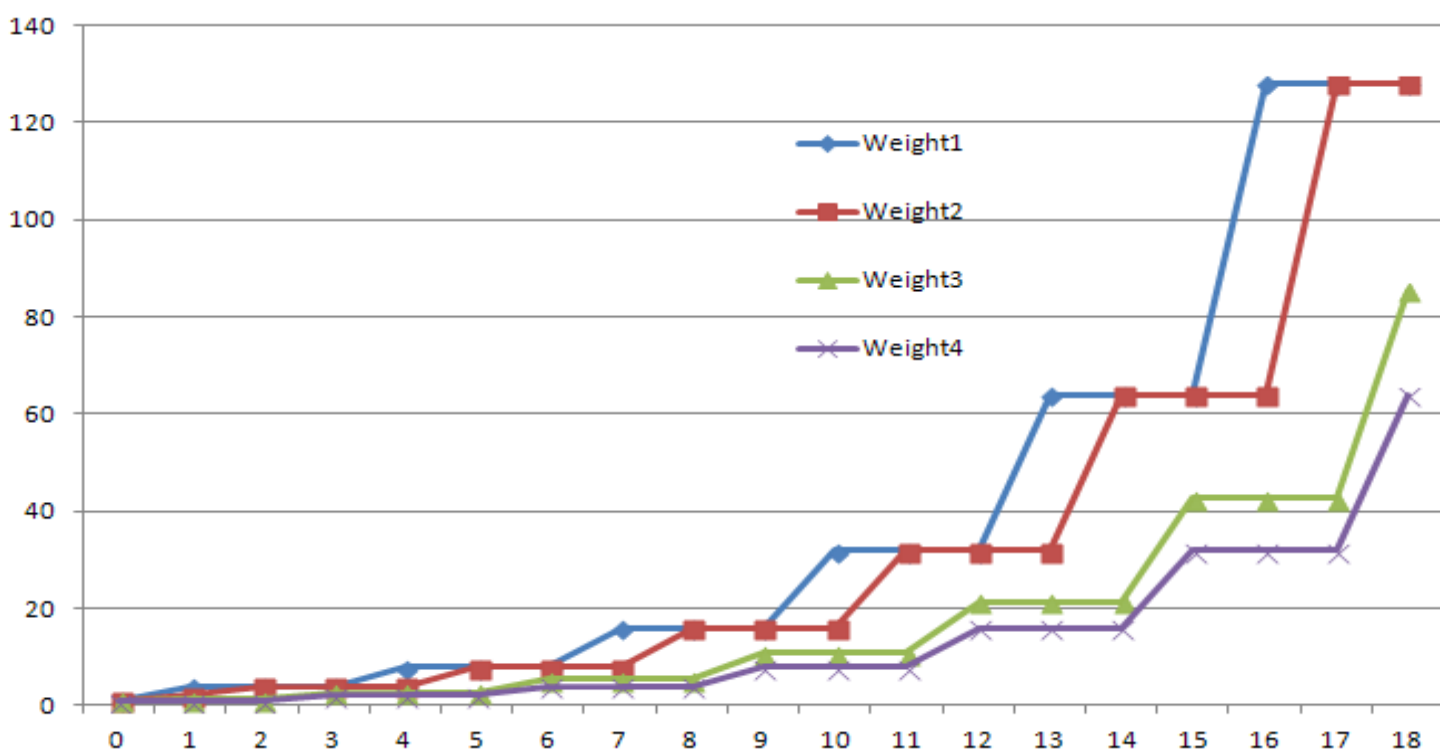

Fig.A7: Dynamic characteristics of the raw priority weight vector for matrix $\mathrm{B}_{2}$.

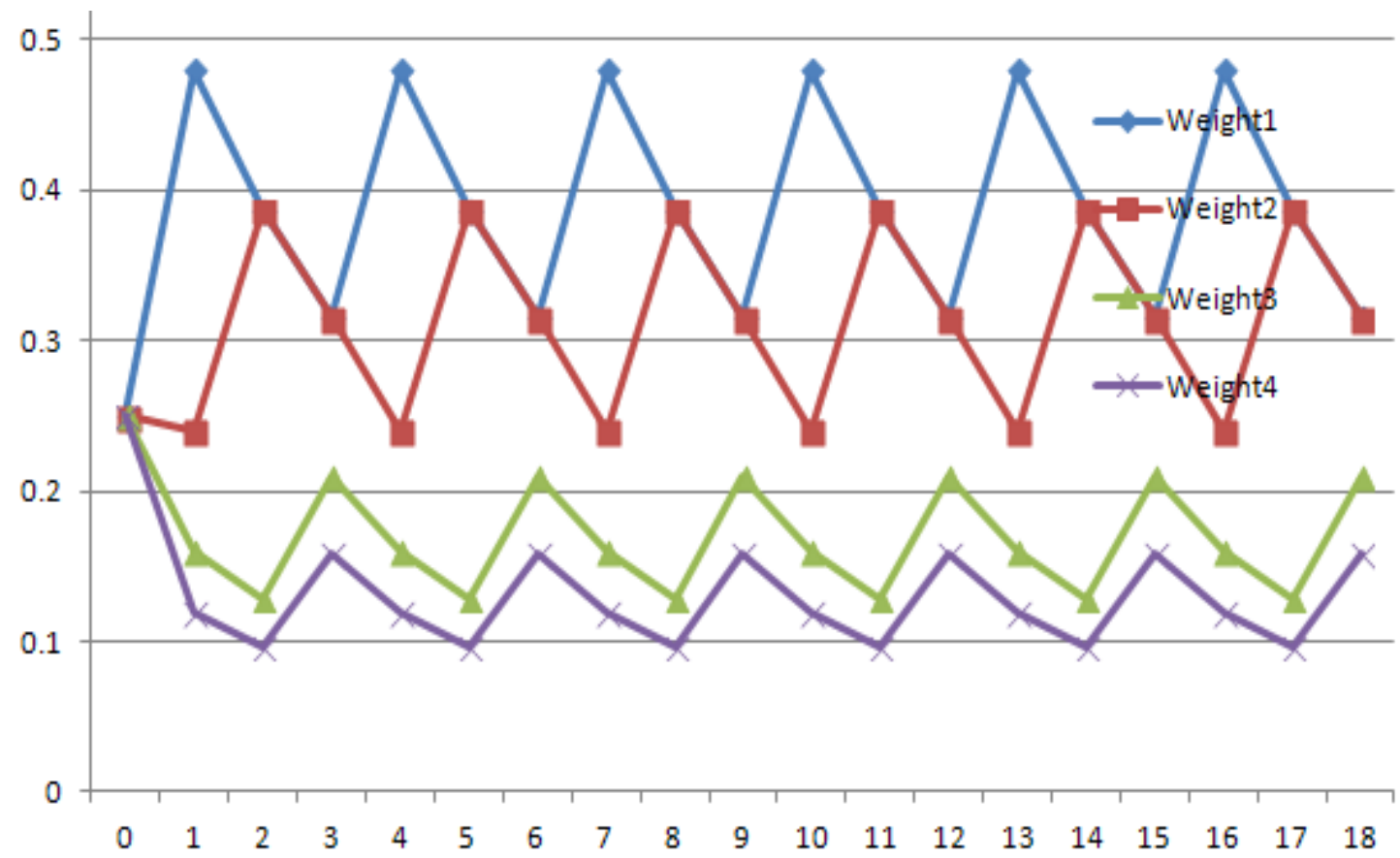

Fig.A8: Dynamic characteristics of the normalized priority weight vector for matrix $\mathrm{B}_{2}$. 